\section{ORIGINAL RESEARCH}

\author{
C. Roth \\ D. Junk
}

P. Papanagiotou

A. Keuler

H. Körner

M. Schumacher

W. Reith

\title{
A Comparison of 2 Stroke Devices: The New Aperio Clot-Removal Device and the Solitaire AB/FR
}

BACKGROUND AND PURPOSE: Several studies have shown promising results for the use of selfexpandable intracranial stents for the treatment of acute ischemic stroke. This new technique combines immediate flow restoration after stent deployment with high recanalization rates. In the present study, the safety and efficacy of the new Aperio clot-removal device was tested in comparison with the Solitaire AB device.

MATERIALS AND METHODS: The experiments were performed in swine with a weight of 35-50 kg following established models. The experimental thrombi were prepared by using a new flow model (Thrombus Loop) to produce radiopaque thrombi. Thrombi were cut into lengths of $10 \mathrm{~mm}$ and injected into the target vessel. The occlusion was verified by DSA and rated with the help of the TICI score. A microcatheter was placed distal to the thrombus. The devices were placed with the proximal third within the thrombus and were retrieved under continuous aspiration in their released state into the guiding sheath. We performed 23 procedures with the Aperio device and 18 procedures with the Solitaire $A B$ and FR devices. Control angiograms were obtained 3 and 5 minutes after device deployment to evaluate the flow restoration. The number of attempts to reach a $\mathrm{TICl} 2 / 3$ score was recorded as well as the time to recanalization and the device-related complications. Vasospasm, vascular perforation, intramural arterial dissection, or embolization of a previously uninvolved territory was defined as a device-related complication.

RESULTS: Defined radiopaque thrombi from whole blood could reliably be created by using the Thrombus Loop. Both devices demonstrated a high recanalization rate of $100 \%(\mathrm{TICl} 3)$ in the target vessel with no device-related complications. No significant differences were found between the 2 devices.

CoNCLUSIONS: In this small study, we could show that radiopaque thrombi of whole blood with a defined diameter could be reliably created by using the Thrombus Loop. The new "stent-retriever" (Aperio System) seems to be a very efficient and safe addition to the existing repertoire of clot-removal devices.

ABBREVIATIONS: $\mathrm{BaSO}_{4}=$ barium sulfate; $\mathrm{mRS}=$ modified Rankin Scale; $\mathrm{TICl}=$ Thrombolysis in Cerebral Infarction score

$\mathrm{n}$ the past few years, interventional treatment of acute ischemic stroke has developed rapidly. Stroke is the third leading cause of death in North America, Europe, and Asia; also it is one of the leading causes of adult disability. The only known drug therapy for acute ischemic stroke is thrombolysis with rtPA. In many clinical trials, treatment with rtPA has proved to be effective in improving the clinical outcome and reducing subsequent disability. However, the advancement of this treatment approach has stalled. The only improvement in this therapeutic regimen has been the extension of the time frame in which it can be safely administered. ${ }^{1-7}$ Treatment of ischemic stroke in patients with a large intracranial vessel occlusion still remains a challenge because IV thrombolysis often reaches its limit. ${ }^{8,9}$ The recanalization rates achieved with IV rtPA for

Received August 16, 2010; accepted after revision October 27

From the Clinic for Diagnostic and Interventional Neuroradiology (C.R., D.J., P.P., H.K., W.R.), Saarland University Hospital, Homburg/Saar, Germany; and Department of Neuroradiology (A.K., M.S.), University Clinic Freiburg, Freiburg, Germany.

C. Roth and D. Junk contributed equally to this work.

Please address correspondence to Christian Roth, Clinic for Diagnostic and Interventional Neuroradiology, Geb. 90.4, Kirrberstr., 66421 Homburg, Germany; e-mail: Christian.roth@ uks.eu

http://dx.doi.org/10.3174/ajnr.A2962 large-vessel arterial occlusion are low, within ranges of $4 \%-$ $32 \%$ depending on the vessel, $4 \%$ for ICA occlusions and $32 \%$ for MCA occlusions. ${ }^{10,11}$

Several trials have shown the efficacy of a mechanical treatment approach in acute stroke caused by large-vessel occlusion. ${ }^{12}$ These trials were able to show that a mechanical approach alone or in combination with IV and intra-arterial rtPA administration could improve the recanalization rate and thereby the outcome of the treated patients. Several reports of the use of intracranial stent placement in acute ischemic stroke have been published. In these trials, a recanalization rate of up to $100 \%$ could be achieved. Recently a new technique by using a self-expendable intracranial stent as a thrombectomy device was introduced. ${ }^{13,14}$ Several studies have shown recanalization rates of up to $90 \%$ (TICI 3 ) and good clinical outcome in $54 \%$ of the patients (mRS $0-2$ ) by using this technique.

In this study, we compared the Solitaire AB/FR stent (ev3, Irvine, California) against the new Aperio device (Acandis, Pforzheim, Germany) in an animal stroke model. The Aperio is a hybrid-design stent-based clot retriever with alternating segments of small closed cells and wider hybrid cells for high 

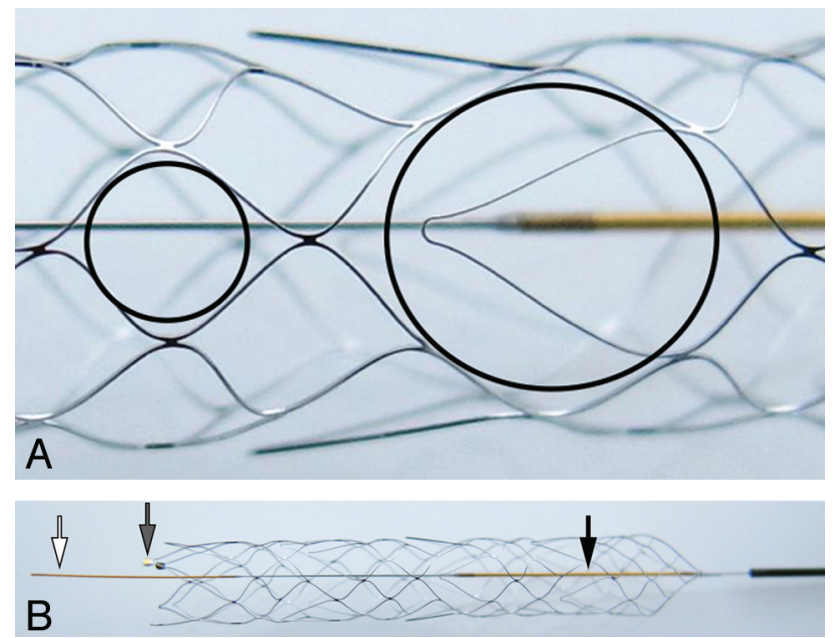

Fig 1. $A$, The small circle shows the smaller closed cells with a diameter of $1.7 \mathrm{~mm}$; the larger circle illustrates the larger hybrid (clot-catching) cells for better thrombus fixation in tortuous vessel segments $(3.7 \mathrm{~mm})$. B, The Aperio device in its unfolded state. The white and black arrows mark the distal and proximal radiopaque wire markers; the gray arrow shows the radiopaque markers at the end of the device, indicating complete device unfolding.

flexibility, improved vessel apposition, and better thrombus fixation during retrieval.

\section{Materials and Methods}

\section{Revascularization Devices}

The Solitaire AB/FR device is a laser-cut self-expanding split-design closed-cell nitinol stent device. It is available with a diameter of $4 \mathrm{~mm}$ (length, 15 or $20 \mathrm{~mm}$ ) or $6 \mathrm{~mm}$ (length, 20 or $30 \mathrm{~mm}$ ) and is delivered through a 0.021 - or 0.027 -inch microcatheter.

The Aperio is a self-expanding nitinol laser-cut stentlike thrombectomy device. It contains 3 distal device markers to indicate device expansion (Fig $1 B$, gray arrow) as well as a proximal and distal wire marker (Fig $1 B$, white and black arrows). The device has a hybrid design with a mixture of closed and partially open cells. The small closed cells (Fig 1A, small black circle) are supposed to optimize vessel adaption; the wider hybrid cells, clot-catching cells (Fig 1A, large black circle), contain an anchoring element for optimal clot fixation in tortuous vessels. It is available with a diameter of $4.5 \mathrm{~mm}$ and a length of $40 \mathrm{~mm}$ for the treatment of vessels ranging from 2 to $4 \mathrm{~mm}$. It is bundled with the 0.027 -inch Acandis microcatheter, which has a length of $1550 \mathrm{~mm}$ and is compatible with 0.010 - to 0.014 -inch guidewires and $6 \mathrm{~F}$ guiding catheters.

\section{Animal Care}

All procedures were conducted according to international guidelines. Eight swine ranging from 35 to $50 \mathrm{~kg}$ were used in this study. Each animal had free access to food and water until the morning of the angiography.

Sedation was induced by a mixture of ketamine (KetaVet, $30 \mathrm{mg} /$ $\mathrm{kg}$ ) and xylazine (TranquiVed, $2.5 \mathrm{mg} / \mathrm{kg}$ ) and atropine $(0.05 \mathrm{mg} / \mathrm{kg})$. Endotracheal intubation was performed. General anesthesia was maintained by IV infusion of thiopental (Trapanal, $2.6-\mathrm{g} / 100 \mathrm{~mL}$ sodium chloride solution, $15-20 \mathrm{~mL} / \mathrm{hr}$ ). After the swine was put under general anesthesia, the femoral artery and vein were exposed surgically and an $8 \mathrm{~F}$ sheath (Avanti+ Introducer, Cordis, Bridgewater, New Jersey) and a 4F (Cordis) sheath, respectively, were introduced into the vessels. As a guiding catheter, an $8 \mathrm{~F}$ balloon catheter (Merci; Concentric
Medical, Mountain View, California) was used. The procedures were performed with the swine under systemic heparinization.

Vital parameters such as arterial blood pressure, heart rate, and expired oxygen and carbon dioxide levels were continuously recorded. The expired oxygen levels were kept at $30 \%$ of the expired vol. After the experiments, the animals were euthanized with an IV injection of pentobarbital.

\section{Angiography}

Selective intra-arterial DSA was performed on a high-resolution angiographic system (Ziehm Vision imaging, Nuremberg, Germany); Ultravist-370 (iopromide; Bayer Schering Pharma, Berlin, Germany) was used for vessel contrast. Selective occlusion of vessels in the subclavian or carotid vasculature within a 2- to $4-\mathrm{mm}$ size range was performed. These vessel regions simulate the anatomic setting of an occlusion of the MCA and the basilar artery in the human circulation. The guiding catheter was pulled back into a larger vessel segment to restore arterial flow. The occluded vessel segment was then navigated with a 0.21 -inch microcatheter (Rebar 18, ev3) for the deployment of the Solitaire device or with the microcatheter that comes with the Aperio device, both in combination with a 0.014-inch microwire (Traxcess; MicroVention, Aliso Viejo, California).

\section{Preparation of the Experimental Thrombi}

The thrombi used for the trial were prepared with a modified method of the Chandler loop, ${ }^{15,16}$ with the help of a dynamic clot-generating system (Thrombus Loop, Acandis). A polyvinyl chloride tubing with an inner diameter of $5 \mathrm{~mm}$ and a length of $50 \mathrm{~cm}$ was filled with $3 \mathrm{~mL}$ of blood from the corresponding swine for the trial. For better fluoroscopic visibility, the blood was mixed with $300 \mathrm{mg}$ of $\mathrm{BaSo}_{4} / 12 \mathrm{~mL}$ of blood. The tubing rotated at a rate of $20 \mathrm{rpm}$ in a water basin at $37^{\circ} \mathrm{C}$. After 15 minutes, the thrombi were drained and rinsed with physiologic saline solution and stored in a basin with the same solution at room temperature for 60 minutes.

\section{Thrombus Application}

After cutting the thrombi in lengths of $10 \mathrm{~mm}$, we injected the thrombi, with help of polytetrafluoroethylene tubing (4-mm inner diameter) with a $20-\mathrm{mL}$ syringe, both filled with physiologic saline solution to avoid air embolism, via a long 6F sheath (Strada; St Jude Medical, St Paul, Minnesota) into the target vessel.

\section{Mechanical Thrombectomy}

An $8 \mathrm{~F}$ balloon catheter (Merci) was advanced into the proximal subclavian artery and the proximal common carotid artery, respectively. The target vessel occlusion was verified angiographically and rated with the help of the TICI scale. ${ }^{17}$ The thrombus was passed with a microcatheter (3F Microcatheter, Acandis; Rebar, ev3) with a 0.014inch microwire (Traxcess; SilverSpeed, ev3). After placement of the microcatheter distal to the thrombus, verified by intra-arterial contrast medium injection, we advanced the device through the microcatheter and placed it with the proximal third within the thrombus. A control angiogram was obtained after successful unfolding of the device to evaluate re-establishment of flow and again after 3 and 5 minutes. Then the balloon at the tip of the guiding catheter was inflated, and the device was pulled back in its unleashed state under continuous aspiration with a $50-\mathrm{mL}$ syringe together with the microcatheter into the guiding sheath. After removal of the device and the microcatheter, another $50 \mathrm{~mL}$ was aspirated from the guiding sheath to prevent re-embolization of a possibly lost clot. If the following control 

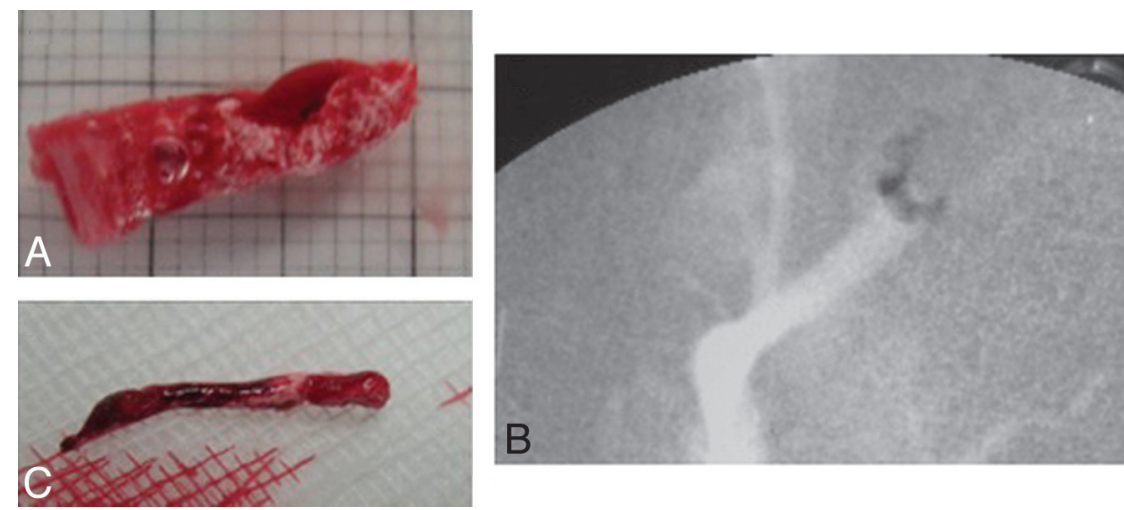

Fig 2. $A$, Thrombus before insertion. $B$, Radiographic image of radiopaque thrombus. $C$, Thrombus after extraction.

angiogram showed a TICI score lower than 2, the procedure was repeated until a TICI score of 2 or 3 was achieved.

\section{Measurement of Outcome}

The time to recanalization, the number of attempts to reach recanalization, device deployment, and the rate of device-related complications were recorded. For device-related complications, we noted vasospasm, vascular perforation, intramural arterial dissection, or embolization of a previously uninvolved territory.

The DSAs were analyzed by 2 experienced neuroradiologists (M.S. and W.R.), who were blinded to the devices used, on a MacPro workstation (Apple, Bothell, Washington) running OsiriX 3.8.1 open-source DICOM software (Pixmeo, Geneva, Switzerland). The angiograms were carefully read for any sign of postinterventional vasospasm, dissection, or vessel perforation. The evaluation of embolization of previously uninvolved vessel territories during the retrieval attempt was done by reading the unsubtracted images for radiopaque clot as well as reading the subtracted images for newly occluded branches.

\section{Results}

A total of 8 swine with a weight of $35-50 \mathrm{~kg}$ were randomized for this study. There were 23 procedures performed with the new Aperio device and 18 procedures, with the Solitaire $\mathrm{AB} / \mathrm{FR}$ device. There were no significant differences in the baseline characteristics between the 2 devices.

With the modified clot-generating procedure, we were able to generate clots similar in size and length with a high radiopacity because of the added $\mathrm{BaSo}_{4}$ (Fig 2).

Both devices demonstrated a very good fluoroscopic visibility and good unfolding of the device after deployment. Furthermore, both devices showed a high recanalization rate of $100 \%$ in the target vessel (with the following average TICI scores: Aperio, TICI 2.8; Solitaire AB/FR, TICI 2.7) (Fig 3) and excellent device performance with no device-related acute thrombosis, vessel dissection, or vessel perforation. Distal embolization was recorded in $8 \%$ of the procedures with the Aperio and in $11 \%$ with the Solitaire AB. Device-related vasospasm was seen in $9 \%$ of the procedures with the Aperio device and in $6 \%$ with the Solitaire AB/FR device. A mean of 1.4 runs was needed with the Aperio device to reach a TICI $\geq 2$ score and 1.2 runs, for the Solitaire, respectively (Table and Fig 3).



Fig 3. Comparison of the Solitaire $A B / F R$ and the Aperio devices regarding acute recanalization and clot removal.

\begin{tabular}{lcc}
\hline Comparison of the 2 devices & & \\
\hline & Aperio & Solitaire \\
\hline No. of attempts, TICI 2/3 & 1.4 & 1.2 \\
TICI 2/3 score & $100 \%$ & $100 \%$ \\
Distal embolization & $8 \%$ & $11 \%$ \\
Vasospasm & $9 \%$ & $6 \%$ \\
Acute thrombosis/dissection/perforation & 0 & 0 \\
\hline
\end{tabular}

\section{Discussion}

Successful recanalization is associated with improved outcome after acute ischemic stroke. ${ }^{18} \mathrm{IV}$ rtPA administration has proved useful up to 4.5 hours after stroke-symptom onset. ${ }^{3}$ However, recanalization rates in large-vessel occlusions are low, within ranges of $4 \%-32 \%$, depending on the vessel, $4 \%$ for ICA occlusions, and $32 \%$ for MCA occlusions. ${ }^{10,11} \mathrm{Re}-$ cently, a new technique by using a self-expandable stent system as a clot-removal device has been introduced. ${ }^{14}$ The first results using this technique show high recanalization rates and a high rate of favorable clinical outcome $(\mathrm{mRS} \leq 2$ in up to $45 \%$ at 90 days $).{ }^{13,19}$

The aim of our study was to compare the safety and efficacy of the new Aperio clot-removal device with the Solitaire $\mathrm{AB} / \mathrm{FR}$ device, which was the device mostly used in the previous studies with this treatment approach.

In this study, immediate flow restoration could be achieved in all cases after device deployment. However, the rate of recanalization decreased with time so that after 5 minutes, the target vessel was almost re-occluded in most of the cases, no matter which device was used. This outcome indicated that there would not be a benefit in leaving the device in place for much longer. This phenomenon might be caused by migra- 
tion of the thrombus into the stent struts. ${ }^{20}$ Both devices were able to reach recanalization rates of $100 \%$ of the target vessel (TICI 2/3). These rates are higher than those in previous clinical studies; however, the vessels presented in this animal model were less tortuous and less elongated and showed no sign of atherosclerotic changes, and the recanalization rates were within the range of those in previous animal studies using a similar model. ${ }^{20}$ No device-related complications occurred regarding vessel dissection or perforation; however, vasospasm was seen in up to $8 \%$ of the cases. This is a common occurrence in this type of animal model. ${ }^{21}$

\section{Limitations}

In this animal model, the vessels used for thrombectomy maneuvers were within the size range of intracranial human vessels; however, the whole vascular system was less tortuous and less elongated and no atherosclerosis was present and the vessels were prone to vasospasm. Because only 1 entity of thrombus was used, different compositions of thrombi might show different results in the treatment with the 2 devices.

\section{Conclusions}

In this small study, we could show that radiopaque thrombi of whole blood with a defined diameter could be reliably created by using the Acandis Thrombus Loop. The new "stent-retriever" (Aperio System) seems to be a very efficient and safe addition to the existing repertoire of clot-removal devices.

Disclosure: Andreas Keuler-RELATED: Provision of Writing Assistance, Medicines, Equipment, or Administrative Support. University Hospital Freiburg, Germany,* Comments: payment by the Acandis company for animal experiments testing the clot retriever. Martin Schumacher-RELATED: Provision of Writing Assistance, Medicines, Equipment, or Administrative Support. University Hospital Freiburg, Germany, ${ }^{*}$ Comments: payment by the Acandis company for animal experiments testing the clot retriever. $\left({ }^{*}\right.$ Money paid to the institution.)

\section{References}

1. Tissue plasminogen activator for acute ischemic stroke: the National Institute of Neurological Disorders and Stroke rt-PA Stroke Study Group. N Engl J Med 1995;333:1581-87

2. Intracerebral hemorrhage after intravenous t-PA therapy for ischemic stroke: the NINDS t-PA Stroke Study Group. Stroke 1997;28:2109-18
3. Hacke W, Kaste M, Bluhmki E, et al. Thrombolysis with alteplase 3 to 4.5 hours after acute ischemic stroke. N Engl J Med 2008;359:1317-29

4. Hacke W, Kaste M, Fieschi C, et al. Randomised double-blind placebo-controlled trial of thrombolytic therapy with intravenous alteplase in acute ischaemic stroke (ECASS II): Second European-Australasian Acute Stroke Study Investigators. Lancet 1998;352:1245-51

5. Steiner T, Bluhmki E, Kaste M, et al. The ECASS 3-hour cohort: secondary analysis of ECASS data by time stratification-ECASS Study Group. European Cooperative Acute Stroke Study. Cerebrovasc Dis 1998;8:198-203

6. Wahlgren N, Ahmed N, Dávalos A, et al. Thrombolysis with alteplase for acute ischaemic stroke in the Safe Implementation of Thrombolysis in Stroke-Monitoring Study (SITS-MOST): an observational study. Lancet 2007;369:275-82

7. Wahlgren N, Ahmed N, Davalos A, et al. Thrombolysis with alteplase 3-4.5 h after acute ischaemic stroke (SITS-ISTR): an observational study. Lancet 2008; 372:1303-09. Epub 2008 Sep 12

8. Schellinger P, Kohrmann M, Hacke W. Thrombolytic therapy for acute stroke. In: Fisher M, ed. Handbook of Clinical Neurology. Vol. 94. Amsterdam, the Netherlands: Elsevier; 2008:1155-93

9. Schellinger P, Ringleb P, Hacke W. European Stroke Organisation 2008 guidelines for managing acute cerebral infarction or transient ischemic attack. Part 2. [in German]. Nervenarzt 2008;79:1180-84, 1186-88, 1190-201

10. Bhatia $\mathrm{R}$, Hill $\mathrm{MD}$, Shobha $\mathrm{N}$, et al. Low rates of acute recanalization with intravenous recombinant tissue plasminogen activator in ischemic stroke: real-world experience and a call for action. Stroke 2010;41:2254-58

11. Tomsick T, Broderick J, Carrozella J, et al. Revascularization results in the Interventional Management of Stroke II trial. AJNR Am J Neuroradiol 2008;29:582-87

12. Furlan A, Higashida R, Wechsler L, et al. Intra-arterial prourokinase for acute ischemic stroke: the PROACT II study-a randomized controlled trial. Prolyse in Acute Cerebral Thromboembolism. JAMA 1999;282:2003-11

13. Roth C, Papanagiotou $P$, Behnke S, et al. Stent-assisted mechanical recanalization for treatment of acute intracerebral artery occlusions. Stroke 2010;41: 2559-67

14. Papanagiotou P, Roth C, Walter S, et al. Treatment of acute cerebral artery occlusion with a fully recoverable intracranial stent: a new technique. $\mathrm{Circu}$ lation 2010;121:2605-06

15. Kan I, Yuki I, Murayama Y, et al. A novel method of thrombus preparation for use in a swine model for evaluation of thrombectomy devices. AJNR Am J Neuroradiol 2010;31:1741-43. Epub 2010 Jan 21

16. Poole JC. A study of artificial thrombi produced by a modification of Chandler's method. Q J Exp Physiol Cogn Med Sci 1959;44:377-84

17. Higashida $\mathrm{RT}$, Furlan $\mathrm{AJ}$, Roberts $\mathrm{H}$, et al. Trial design and reporting standards for intra-arterial cerebral thrombolysis for acute ischemic stroke. Stroke 2003;34:e109-37

18. Rha JH, Saver JL. The impact of recanalization on ischemic stroke outcome: a meta-analysis. Stroke 2007;38:967-73

19. Castaño C, Dorado L, Guerrero C, et al. Mechanical thrombectomy with the Solitaire $\mathrm{AB}$ device in large artery occlusions of the anterior circulation: a pilot study. Stroke 2010;41:1836-40

20. Mordasini P, Frabetti N, Gralla J, et al. In vivo evaluation of the first dedicated combined flow-restoration and mechanical thrombectomy device in a swine model of acute vessel occlusion. AJNR Am J Neuroradiol 2011;32:294-300

21. Gralla J, Schroth G, Remonda L, et al. A dedicated animal model for mechanical thrombectomy in acute stroke. AJNR Am J Neuroradiol 2006;27:1357-61 